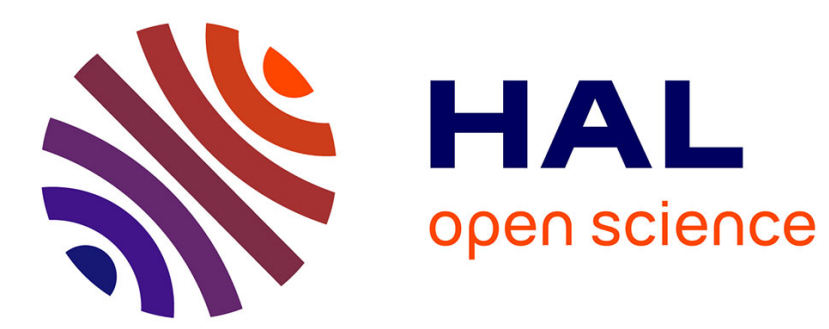

\title{
Un théorème de Torelli infinitésimal à coefficients
}

Damien Mégy

\section{- To cite this version:}

Damien Mégy. Un théorème de Torelli infinitésimal à coefficients. 2010. hal-00502399

\section{HAL Id: hal-00502399 \\ https://hal.science/hal-00502399}

Preprint submitted on 28 Jul 2010

HAL is a multi-disciplinary open access archive for the deposit and dissemination of scientific research documents, whether they are published or not. The documents may come from teaching and research institutions in France or abroad, or from public or private research centers.
L'archive ouverte pluridisciplinaire HAL, est destinée au dépôt et à la diffusion de documents scientifiques de niveau recherche, publiés ou non, émanant des établissements d'enseignement et de recherche français ou étrangers, des laboratoires publics ou privés. 


\title{
Un théorème de Torelli infinitésimal à coefficients
}

\author{
Damien Mégy a \\ ${ }^{\mathrm{a}}$ Institut Fourier, 100 rue des maths, BP 74, 38402 St Martin d'Hères cedex, France
}

\section{Résumé}

On généralise un théorème de Torelli infinitésimal pour des hypersurfaces de haut degré de M. Green [Gre85] dans le cas de coefficients tordus.

\section{Abstract}

An infinitesimal Torelli theorem with twisted coefficients We generalize an infinitesimal Torelli theorem for hypersurfaces of high degree of M. Green [Gre85], for twisted coefficients.

Je remercie Philippe Eyssidieux, ainsi que le rapporteur, pour m'avoir aidé à améliorer cette note.

Soit $Y$ une variété complexe projective lisse de dimension $n+1$, plongée dans un espace projectif $\mathbb{P}$, linéairement normale. On note $L$ le fibré $\mathscr{O}_{Y}(1), \mathbb{P}^{\vee}$ l'espace projectif dual de $\mathbb{P}$, et $\mathfrak{Y}:=\{(y, H) \in$ $\left.Y \times \mathbb{P}^{\vee}, y \in H\right\}$ la section hyperplane universelle de $Y$, munie des projections $\pi$ et $a$ vers $\mathbb{P}^{\vee}$ et $Y$.

Soit $\mathbb{W}$ une variation de structure de Hodge complexe polarisée (VSH) de poids $w$ sur $Y$, avec $W^{w, 0} \neq 0$. On note $\mathbb{V}$ la VSH sur l'ouvert des valeurs régulières de $\pi$ définie par $\mathbb{V}=R^{n} \pi_{*}\left(a^{*} \mathbb{W}\right)$.

Théorème 1 - Si L est suffisamment ample, l'application des périodes de $\mathbb{V}$ a un rang supérieur ou égal à celui de l'application de Kodaira-Spencer de la famille de sections hyperplanes.

Remarquons que si de plus $H^{0}\left(X, T_{X}\right)=0$, alors l'application de Kodaira-Spencer est injective.

Lorsque $\mathbb{W}=\mathbb{C}_{Y}$, la dérivée de l'application des périodes se factorise par l'application de KodairaSpencer de la famille d'hypersurfaces, et le second facteur est un morphisme de cup-produit par la classe de Kodaira-Spencer ([Gri68]). Ce morphisme est injectif par le théorème de Torelli infinitésimal de M. Green [Gre85], theorem 0.1 p. 135, si $L$ est suffisamment ample, ce dernier résultat étant une généralisation du théorème de Torelli infinitésimal de Griffiths [Gri69] pour les hypersurfaces de degré $\geq n+2$ de $\mathbb{P}^{n+1}$.

Email address: damien.megy@ujf-grenoble.fr (Damien Mégy). 
Le théorème 1 découle des deux résultats suivants, qui utilisent des notations indépendantes :

Proposition 2 - Soit $\pi: X \rightarrow S$ un morphisme projectif lisse entre variétés complexes, de dimension relative $n$. Si $s \in S$, on note $X_{s}$ la fibre de $\pi$ au-dessus de $s$, et $\rho_{s}$ l'application de Kodaira-Spencer en $s$. Soit $\mathbb{W}$ une VSH complexe polarisée de poids w sur $X, \mathbb{V}=R^{n} \pi_{*} \mathbb{W}$, qui est une VSH de poids $n+w$ sur $S$, et $V^{p, q}$ les fibrés de Hodge de $\mathbb{V}$. Soit enfin $\mathscr{P}^{n+w}$ la dernière composante de l'application des périodes de $\mathbb{V}$, qui à un point $s \in S$ associe $V_{s}^{n+w, 0} \subset V_{s}$. Alors, il existe un diagramme commutatif

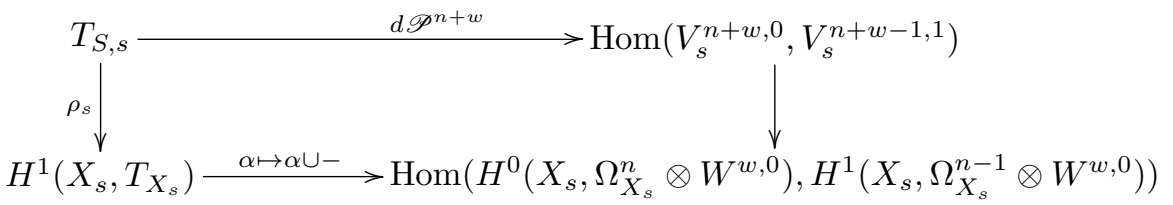

Proposition 3 - Soit $Y$ une variété projective lisse de dimension $n+1>2, F$ un fibré vectoriel holomorphe sur $Y, L$ un fibré en droites holomorphe très ample sur $Y$. Quitte à remplacer $L$ par $L^{\otimes k}$, pour toute section globale $s$ de $L$ dont le lieu d'annulation $Z \subset Y$ est lisse, le morphisme de cup-produit

$$
H^{1}\left(Z, T_{Z}\right) \rightarrow H^{0}\left(Z, K_{Z} \otimes F\right)^{*} \otimes H^{1}\left(Z, \Omega_{Z}^{n-1} \otimes F\right)
$$

est injectif.

Cette dernière proposition est une version légèrement plus générale de [Gre85], theorem 0.1, et se démontre de manière similaire. Nous donnons ici une preuve de la proposition 2.

Démonstration de la proposition 2 - Commençons par construire la flèche

$$
\operatorname{Hom}\left(V_{s}^{n+w, 0}, V_{s}^{n+w-1,1}\right) \rightarrow \operatorname{Hom}\left(H^{0}\left(X_{s}, \Omega_{X_{s}}^{n} \otimes W^{w, 0}\right), H^{1}\left(X_{0}, \Omega_{X_{s}}^{n-1} \otimes W^{w, 0}\right)\right) .
$$

Soit $W$ le fibré différentiable sous-jacent à $\mathbb{W}$. Écrivons sa connexion plate $D$ sous la forme $D=$ $\underbrace{\partial+\bar{\theta}}_{D^{\prime}}+\underbrace{\bar{\partial}+\theta}_{D^{\prime \prime}}$, avec les notations de [Sim92] :

$$
\begin{gathered}
\partial\left(\mathscr{A}^{r, s}\left(W^{p, q}\right) \subset \mathscr{A}^{r+1, s}\left(W^{p, q}\right),\right. \\
\bar{\partial}\left(\mathscr{A}^{r, s}\left(W^{p, q}\right) \subset \mathscr{A}^{r, s+1}\left(W^{p, q}\right),\right. \\
\theta\left(\mathscr{A}^{r, s}\left(W^{p, q}\right) \subset \mathscr{A}^{r+1, s}\left(W^{p-1, q+1}\right),\right. \\
\bar{\theta}\left(\mathscr{A}^{r, s}\left(W^{p, q}\right) \subset \mathscr{A}^{r, s+1}\left(W^{p+1, q-1}\right) .\right.
\end{gathered}
$$

Soit $\mathscr{A}(W)^{P, Q}=\bigoplus_{p+r=P, s+q=Q} \mathscr{A}^{r, s}\left(W^{p, q}\right)$. Alors $D^{\prime}$ est de type $(1,0)$ et $D^{\prime \prime}$ de type $(0,1)$. De plus, on a $D^{\prime \prime 2}=0$.

On peut définir la même bigraduation sur $X_{s}$, par restriction. Soit $K_{P, Q}^{\bullet}$ le complexe $K_{P, Q}^{i}=\mathscr{A}_{X_{s}}(W)^{P, Q-n+i}$, muni de la différentielle $D^{\prime \prime}$. Deligne a montré (voir [Zuc79]) que $H^{n}\left(X_{s}, \mathbb{W}\right.$ ) admet une structure de Hodge

$$
H^{n}\left(X_{s}, \mathbb{W}\right)=\bigoplus_{P+Q=n+w} H^{n}\left(X_{s}, \mathbb{W}\right)^{P, Q},
$$

avec

$$
H^{n}\left(X_{s}, \mathbb{W}\right)^{P, Q}:=\mathbb{H}^{n}\left(X_{s}, K_{P, Q}^{\bullet}\right) .
$$


De plus, le complexe $K_{n+w, 0}^{\bullet}[n]$ coïncide avec la résolution de Dolbeault de $\Omega_{X_{s}}^{n} \otimes W^{w, 0}$, donc on a un isomorphisme

$$
V_{s}^{n+w, 0}:=H^{n}\left(X_{s}, \mathbb{W}\right)^{n+w, 0} \simeq H^{0}\left(X_{s}, \Omega_{X_{s}}^{n} \otimes W^{w, 0}\right) .
$$

Enfin, le morphisme de complexes

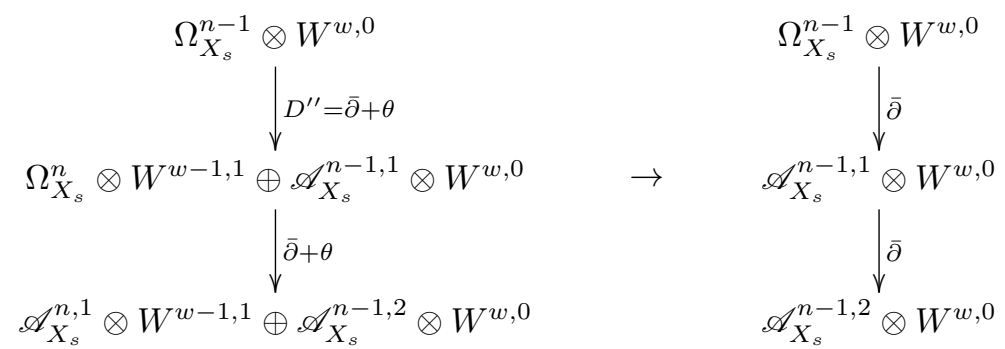

donne en hypercohomologie un morphisme

$$
V_{s}^{n+w-1,1}:=H^{n}\left(X_{s}, \mathbb{W}\right)^{n+w-1,1} \rightarrow \mathbb{H}^{n}\left(X_{s}, \mathscr{A}^{n-1, \bullet}\left(W^{w, 0}\right)[1-n]\right) \simeq H^{1}\left(X_{s}, \Omega^{n-1} \otimes W^{w, 0}\right) .
$$

Les morphismes (1) et (2) donnent par composition une flèche

$$
\operatorname{Hom}\left(V_{s}^{n+w, 0}, V_{s}^{n+w-1,1}\right) \rightarrow \operatorname{Hom}\left(H^{0}\left(X_{s}, \Omega_{X_{s}}^{n} \otimes W^{w, 0}\right), H^{1}\left(X_{s}, \Omega_{X_{s}}^{n-1} \otimes W^{w, 0}\right)\right) .
$$

En composant à droite par $d \mathscr{P}^{n+w}$, on obtient un diagramme

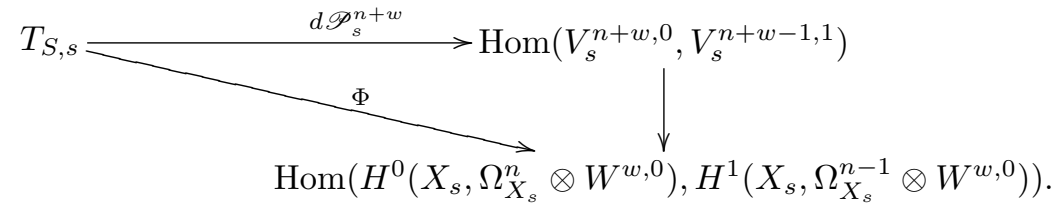

Montrons que ce diagramme se complète en

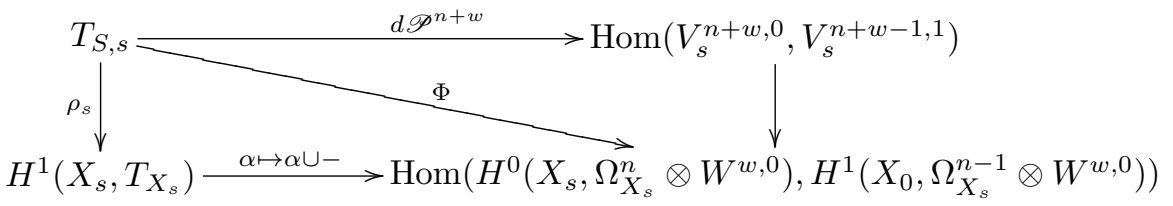

Il s'agit donc de montrer que pour tout $u \in T_{S, s}$, l'endomorphisme $\Phi(u)$ est le cup-produit $\rho_{s}(u) \cup-$.

La preuve, qui repose sur un calcul explicite de dérivée de Lie, est essentiellement la même que celle de Griffiths [Gri68] dans le cas classique. Soit $\sigma \in H^{0}\left(X_{s}, \Omega_{X_{s}}^{n} \otimes W^{w, 0}\right), \tilde{u}$ un champ de vecteurs de type $(1,0)$ sur $X$ défini au voisinage de $X_{s}$ et tel que $\pi_{*} \tilde{u}_{\mid X_{s}}=u$, et $\tilde{\sigma}$ une $n$-forme sur $X$ à valeurs dans $W^{w, 0}$, définie au voisinage de $X_{s}$, dont la restriction aux fibres proches de $X_{s}$ est $D$-fermée. On suppose aussi que la classe de la composante de type $(n+w, 0)$ de $\tilde{\sigma}_{\mid X_{s}}$ est égale à $\sigma$. Alors, on a

$$
d \mathscr{P}^{n+w}(u)(\sigma)=\left[\left(\left.\operatorname{int}(\tilde{u})(D \tilde{\sigma})\right|_{X_{s}}\right)^{n+w-1,1}\right] .
$$

La composition par $H^{n}\left(X_{s}, \mathbb{W}\right)^{n+w-1,1} \rightarrow H^{1}\left(\Omega_{X_{s}}^{n-1} \otimes W^{w, 0}\right)$ consiste à projeter les représentants dans $\mathscr{A}_{X_{s}}^{n-1,1} \otimes W^{w, 0}$ avant de prendre leur classe de Dolbeault classique (pour $\bar{\partial}$ ). En écrivant $D=\partial+\bar{\partial}+\theta+\bar{\theta}$, 
on voit que l'image de $\left[\left(\operatorname{int}(\tilde{u})(D \tilde{\sigma})_{\mid X_{s}}\right)^{n+w-1,1}\right]$ dans $H^{1}\left(\Omega_{X_{s}}^{n-1} \otimes W^{w, 0}\right)$ est pour des raisons de type $\left[\operatorname{int}(\tilde{u})\left(\bar{\partial} \tilde{\sigma}^{n+w, 0}\right)_{\mid X_{s}}\right]$. Donc

$$
\Phi(u)(\sigma)=\left[\operatorname{int}(\tilde{u})\left(\bar{\partial} \tilde{\sigma}^{n+w, 0}\right)_{\mid X_{s}}\right] .
$$

Soit $\operatorname{int}(\bar{\partial} \tilde{u})(-)$ l'opérateur qui combine le produit extérieur pour la partie covariante et la contraction pour la partie contravariante. En dérivant terme à terme, on obtient

$$
\bar{\partial}\left(\operatorname{int}(v)\left(\tilde{\sigma}^{n+w, 0}\right)\right)=\operatorname{int}(\bar{\partial} \tilde{u})\left(\tilde{\sigma}^{n+w, 0}\right)-\operatorname{int}(\tilde{u})\left(\bar{\partial} \tilde{\sigma}^{n+w, 0}\right) .
$$

Ceci implique l'égalité de classes de Dolbeault $\left[\operatorname{int}(\tilde{u})\left(\bar{\partial} \tilde{\sigma}^{n+w, 0}\right)_{\mid X_{s}}\right]=\left[\operatorname{int}(\bar{\partial} \tilde{u})\left(\tilde{\sigma}^{n+w, 0}\right)_{\mid X_{s}}\right]$. Enfin, $\left.\bar{\partial} \tilde{u}\right|_{X_{s}}$ est précisément un représentant de Dolbeault de la classe de Kodaira-Spencer $\rho_{s}(u)$ du vecteur $u$. On a donc :

$$
\Phi(u)(\sigma)=\left[\operatorname{int}\left(\left.\bar{\partial} \tilde{u}\right|_{X_{s}}\right)\left(\tilde{\sigma}_{\mid X_{s}}^{n+w, 0}\right)\right]=\rho_{s}(u) \cup\left[\tilde{\sigma}_{\mid X_{s}}^{n+w, 0}\right]=\rho_{s}(u) \cup \sigma .
$$

Ceci termine la preuve de la proposition.

\section{Références}

[Gre85] M. Green, The period map for hypersurface sections of high degre of an arbitrary variety, Compositio Math. 55 (1985), no. 2, p. 135-156.

[Gri68] P. Griffiths, Periods of integrals on algebraic manifolds, II. (Local study of the period mapping) Amer. J. Math. 90 (1968) p. 805-865.

[Gri69] P. Griffiths, On the periods of certain rational integrals, I, Ann. of Math. 90 (1969), p. 460-495.

[Sim92] C. Simpson, Higgs bundles and local systems, Publ. Math. IHES 75 (1992) p. 5-95

[Zuc79] S. Zucker, Hodge theory with degenerating coefficients : $L^{2}$-cohomology in the Poincaré metric, Ann. Math. 109 (1979), p. 415-476. 